\title{
Economic Burden of COPD by Disease Severity - A Nationwide Cohort Study in Denmark
}

This article was published in the following Dove Press journal:

International Journal of Chronic Obstructive Pulmonary Disease

\author{
Anders Løkke (iD) 1,2 \\ Peter Lange iD $^{3,4}$ \\ Jesper Lykkegaard (iD ${ }^{5}$ \\ Rikke Ibsen ${ }^{6}$ \\ Maria Andersson ${ }^{7}$ \\ Sofie de Fine Licht $\left(\mathbb{D}^{8}\right.$ \\ Ole Hilberg ${ }^{1,2}$

\begin{abstract}
'Department of Medicine, Little Belt Hospital, Vejle, Denmark; ${ }^{2}$ Department of Regional Health Research, University of Southern Denmark, Odense, Denmark; ${ }^{3}$ Medical Department, Copenhagen University Hospital-Herlev, Herlev, Denmark; ${ }^{4}$ Department of Public Health, Section of Epidemiology, University of Copenhagen, Copenhagen, Denmark; ${ }^{5}$ Research Unit for General Practice, Institute of Public Health, University of Southern Denmark, Odense, Denmark; ${ }^{6}$ i2Minds, Aarhus, Denmark; ${ }^{7}$ AstraZeneca Nordic, IVS \& Health Economics, Södertälje, Sweden; ${ }^{8}$ AstraZeneca Nordic,Medical \&
\end{abstract} \\ Regulatory, Södertälje, Sweden
}

Background: Chronic Obstructive Pulmonary Disease (COPD) carries a considerable economic burden, both for individuals and societies. This study aimed to assess direct and indirect costs associated with COPD, and how costs vary across disease severity.

Methods: This was a nationwide, population-based cohort study utilizing Danish health registries. Patients; $\geq 40$ years of age, with an in- and/or outpatient diagnosis of COPD (ICD10 J44) in 2008-2016, were identified in the nationwide Danish COPD Registry. Included patients were matched 1:4 to a population-based non-COPD reference population of 196,623 individuals by sex, year of birth, co-habitation status, and municipality. Patients were grouped by disease severity according to different characteristics including GOLD groups A-D, based on moderate (short-term oral corticosteroid use), presence of severe exacerbations (emergency visit or hospitalization) and symptom score. Index was the date of the first outpatient visit with a symptom score registration. The costs were calculated during a 12 months post-index follow-up.

Results: In all, 49,826 patients with COPD (mean age 69.2 years, 52\% females) were included. Total annual costs, including direct costs, costs for elderly care, and costs for retirement home, were higher for patients with COPD $(€ 28,969)$ compared with the reference population (€10,6913). In GOLD groups A-D, the total direct costs were A: $€ 8,766, \mathrm{~B}: € 13,060, \mathrm{C}$ : $€ 11,113$, and $\mathrm{D}: € 17,749$, respectively. A major driver of direct costs was severe exacerbations. The mean costs per moderate and severe exacerbation were $€ 888$ and $€ 7,091$, respectively, during 28 days of follow-up. The costs for non-COPD-related Health Care Resource Utilization were higher than the COPD-related costs in GOLD groups A-C, but not in GOLD group D.

Conclusion: In this nationwide real-world study, total direct costs were three-fold higher among patients with COPD compared with the reference population. Severe exacerbations were a major driver of the direct costs. The costs increased with increasing disease severity. Keywords: COPD, exacerbations, symptoms, cost, GOLD

\section{Introduction}

Chronic Obstructive Pulmonary Disease (COPD) carries a substantial economic burden - both on an individual and at a societal level. The direct costs of treatment and care for COPD are mainly composed of costs of exacerbations, hospitalizations, outpatient follow-up, visits at the general practitioner, pharmaceutical interventions, laboratory testing, rehabilitation, etc., whereas indirect costs are mainly related to the loss of productivity such as benefits for incapacity to work due to disability, unemployment benefits and social transfer payments. Longitudinal studies describing the economic impact of COPD are scarce. Furthermore, most studies involving cost related to COPD are based on direct costs, such as costs of treatment of COPD, as information about indirect costs is generally not available.
Correspondence: Anders Løkke University of Southern Denmark, Odense, Denmark

Email Aloekke@gmail.com
International Journal of Chronic Obstructive Pulmonary Disease 2021:16 603-613

603

DovePress if in


In the European Union, the total direct costs of respiratory diseases are estimated to constitute $6 \%$ of the total healthcare expenses, with COPD accounting for more than half of these costs ( $56 \%$ - equaling 38.6 billion Euro) ${ }^{1,2}$ In the United States, the estimated direct costs of COPD are $\$ 32$ billion and the indirect costs $\$ 20.4$ billion. $^{3}$

Due to the high prevalence of smoking in Denmark, the prevalence of COPD is high with 270,000 Danish residents with clinically important COPD (GOLD B-D) in a population of 5.7 million in total. ${ }^{4}$ Accordingly, COPDrelated health contacts, use of medication and socioeconomic costs in Denmark are substantial. ${ }^{5-7}$ This has also been shown in a Swedish cohort study, ${ }^{8}$ where hospitalizations with COPD and also COPD-related comorbidities were associated with considerable costs.

Generally, exacerbations seem to be the main cost driver in COPD and the economic burden of exacerbations in COPD has been documented in a number of studies. ${ }^{9-12}$ Although, daily symptom burden is associated with increased risk of exacerbations and hospital admissions, the link between daily dyspnea symptoms and costs has not previously been explored in a longitudinal study. Furthermore, important cost information in an elderly population such as cost of elder care and retirement homes has rarely been described in studies as this information is often not available.

In the present study, we hypothesized that in patients with COPD, both symptom burden and exacerbations are driving direct as well as indirect costs. Using the nationwide Danish COPD Registry (DrCOPD) we assessed the direct and indirect costs associated with COPD in Denmark and examined how these costs vary across disease severity (stratified on Global Initiative for Chronic Obstructive Lung Disease $^{13}$ (GOLD) groups A-D).

\section{Methods}

\section{Study Design and Data Sources}

This was a population-based cohort study using various comprehensive national Danish health registries: (a) the National Hospital Registry covering all inpatient, nonpsychiatric hospitalizations in Denmark since 1977 and all outpatient visits since $1995 .{ }^{14}$ Registration is mandatory and submitted by the treating physician; (b) the National COPD Registry including all COPD patients followed in outpatient specialist clinics in Denmark since $2008 ;^{15}$ (c) the National Prescription Registry including all prescriptions redeemed at pharmacies since 1995 using anatomical therapeutic chemical (ATC) $\operatorname{codes}^{16}$ (d) the National Income Statistics Registry; ${ }^{17}$ and (e) the Civil Registration System for information on vital status, migration and cohabitation. ${ }^{18}$ The accurate individual linkage between the registries was possible by using the unique 10-digit personal identification number, assigned to all Danish residents at birth. Data linkage was performed by Statistics Denmark and the data was anonymized before made available for analyses.

\section{Study Populations}

In the COPD Registry, we identified all patients $\geq 40$ years with a visit in a specialized hospital-based clinic for COPD in 2008-2016 ( $\mathrm{n}=77,156)$. Patients with missing information on Medical Research Council (MRC) dyspnea or incomplete registrations of clinical outcome measures in the COPD registry were excluded as were patients without matching comparisons available $(n=27,330)$. This resulted in a cohort of 49,826 COPD patients included for analyses (Figure 1).

To measure healthcare use and cost in the general population, a population-based reference population of 196,623 individuals without a COPD diagnosis was extracted from the national databases and matched to the COPD patients 1:4 by sex, birth year, co-habitation status, and municipality on the date of the first diagnosis of COPD in the study period.

\section{Baseline COPD Exacerbations and Dyspnea Symptom Scores}

To measure the severity of COPD, the number and severity of COPD exacerbations in the 12 months prior to index date were identified (Supplementary Figure 1). Moderate exacerbations were captured using the Prescription Registry and defined as short-term use of oral corticosteroids (OCS) (corresponding to redeeming a prescription on a maximum of 20 defined daily doses (DDD) of $25 \mathrm{mg}$ prednisolone Anatomical Therapeutic Chemical Classification System (ATC) [code H02AB06] or prednisone [H02AB07]). If patients collected more than one package of OCS on the same day, it was only counted as one exacerbation (and not included as an additional exacerbation).

Severe exacerbations were identified in the Hospital Registry and defined as hospitalizations or emergency room (ER) visits with ICD-10 code J40-44 as primary diagnosis or J13-18 (pneumonia) or J96 (respiratory 


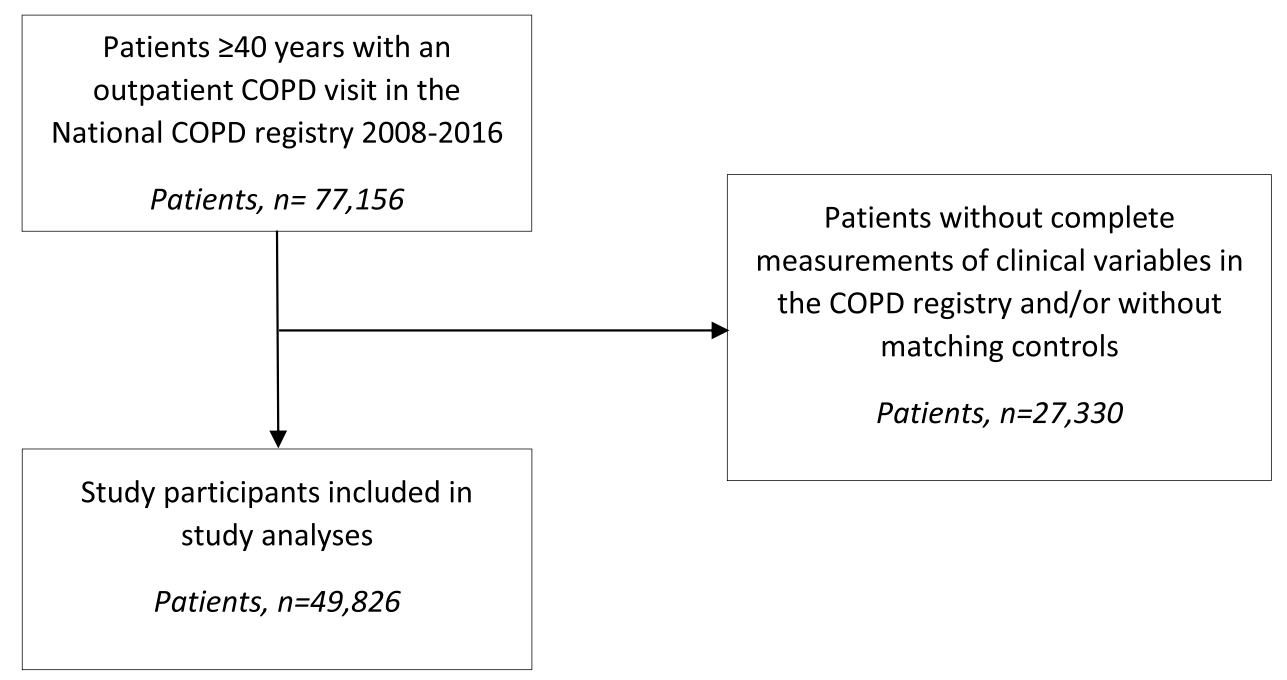

Figure I Diagram showing flow of study participants and final study cohort size.

insufficiency) as primary diagnosis in combination with a secondary diagnosis J40-44. Recurrent exacerbations within 28 days were combined and considered as one exacerbation. Patients with both a moderate and a severe exacerbation within 28 days were counted as having a severe exacerbation with the start date of the exacerbation that occurred first.

Information on dyspnea was measured by the treating physician or nurse using the MRC dyspnea scale ${ }^{19}$ and recorded in the National COPD Registry with a score ranging from 1 (not troubled by breathlessness except on strenuous exercise) to 5 (too breathless to leave the house, or breathless when dressing and undressing). Combining baseline exacerbations and MRC scores, patients were classified into four GOLD groups A-D at baseline (Supplementary Table 1).

\section{Health Care Resource Utilization (HCRU) and Costs}

In this study, both direct and indirect costs as well as costs for elderly care were assessed. Direct costs included acquisition costs for drugs, health care visits in the primary sector, outpatient hospital visits including ER visits and hospital admissions. The costs were captured through Diagnosis-Related Groups (DRG)/Danish Ambulant Grouping System (DAGS) codes and cost of medication collected at the pharmacy from the Prescription Registry.

The indirect costs constituted the difference in earnings between COPD patients and the reference population, based on information on earned income and various social security compensation (eg, pension, early retirement and sick leave payments). In addition, the elderly care costs including costs of elderly care and retirement home were described.

Mean exacerbation costs for both moderate and severe exacerbations were calculated based on HCRU during 28 days after the start of the exacerbation event. COPDrelated costs were defined as inpatient, outpatient, and ER costs related to events with the primary diagnosis with ICD10 codes J44, J96, and J13-J18. All other costs were defined as non-COPD-related costs. Consequently, patients in the reference population could have COPDrelated costs if the primary diagnosis for the contact was J96 or J13-J18, but not J44.

COPD-related medication costs included the cost of respiratory medicine (ATC, R03).

All costs were reported in euro $(€)$ based on the pegged exchange rate DKK7.45/€1.

\section{Statistical Analyses}

Baseline characteristics were described during the baseline year or at the date of the first MRC measurement (index date) (Supplementary Figure 1).

Follow-up for HCRU and costs started on the day after the index date between January 1, 2008, and December 31, 2016. Follow-up ended on the date of death, emigration, 12 months after index date or end of study Dec 31, 2017, whichever occurred first. If a patient had several MRC measurements during the study period only the first measurement was used. Costs were compared between the four GOLD groups using a regression model for each cost with 
age, sex, cohabitation status and the four GOLD groups as covariates with GOLD group A as reference.

cost $=\mathrm{a}^{*}$ age $+\mathrm{b} *$ female $+\mathrm{c}^{*}$ spouse $+\mathrm{d}^{*}$ GoldB + $d^{*}$ GoldC $+d^{*}$ GoldD

A two-step model was used to analyze costs, because health care expenditure was right skewed with a large share of zeros due to many patients having no or low health care expenditure. ${ }^{20}$ In the first step, a probit model estimated the probability that an individual received any health care services in a given year, ie, had positive health care expenditures. In the second step, a gamma-distributed generalized linear model (GLM) was estimated where only individuals who actually received health care services in a given year were included. The GLM model consisted of a gamma-distribution for the expenditures and a log-link function assuming that the logarithm of the expected value of the expenditures could be modelled by a linear combination of parameters on the right-hand side.

In the following, the dependent variable $y_{\text {it }}$ was thus equal to $\operatorname{Pr}($ Expenditures $>0)$ in the first part and $L n$ (Expenditures) in the second part. The right-hand covariates were the same in both the probit specification and the GLM specification. The models were estimated using maximum likelihood estimation. To make interpretation easier and to enable comparison of cost between GOLD groups, we predicted the cost of a 69-year old man with a spouse in each of the four GOLD groups.

The analysis comparing costs between COPD patients and their individually matched population comparisons was calculated for each GOLD group and for all groups combined. The p-values were calculated using bootstrapped $t$-test. For individuals that died during the 12 months follow-up, the costs were weighed by the share of the year they were alive. If, for example, a patient with COPD died, costs were still included for the controls and vice versa as all individuals alive carry costs.

Total costs were estimated as the sum of total direct costs and elderly care costs. Indirect costs, here defined as foregone-earnings, were estimated as the mean income of the reference population minus the mean income of the COPD patients. Both direct, indirect, and elderly care costs were calculated for each GOLD group.

To estimate the mean cost of a moderate and severe exacerbation, all primary care, pharmaceutical, and hospital cost during the 28 days following the exacerbation onset were summarized, including costs on the start date of the exacerbation. In order to ensure that total cost covered 28 days post exacerbation, exacerbations with less than 28 days follow-up for cost due to end of followup $(n=4004)$ or death of the patient $(n=2956)$ were not included. The mean exacerbation cost per patient was calculated by multiplying the mean exacerbation cost (for moderate/severe exacerbations, respectively) with the mean number of exacerbations per GOLD group and divided by the number of patients in the GOLD group.

A sensitivity analysis was conducted to investigate the direct costs per GOLD group when redeemed antibiotics were included in the definition of exacerbations. The ATC codes included in the definition were amoxicillin and enzyme inhibitor (J01CR02), amoxicillin (J01CA04), pivampicillin (J01CA02), phenoxymethylpenicillin (J01CE02) and various macrolides (J01FA01, J01FA06, J01FA09, J01FA10).

\section{Results}

\section{Patient Demographics}

A total of 49,826 patients with COPD and 196,623 matched comparisons in the reference population were included in the study. Baseline characteristics of COPD patients are presented in Table 1. The overall mean age was 69.2 years with the lowest mean age in GOLD group A (65.9 years) and the highest in GOLD D (71.6 years). In total, $52 \%$ of the COPD cohort were females with the largest proportion of females in GOLD group D (55.8\%). The percentage of COPD patients that were cohabitating decreased with COPD severity (from GOLD A to GOLD D).

The MRC scores at baseline followed a normal distribution between the five MRC groups 1 to 5 (Table 1). In GOLD group A and $\mathrm{B}, 10 \%$ and $15 \%$ of the patients, respectively, had experienced a moderate exacerbation at baseline. In GOLD group C and D, severe exacerbations were more frequent than moderate exacerbations during the baseline year prior to index.

The annual exacerbation rate was higher in the follow-up year than in the baseline year for GOLD groups $A$ and B (Table 2), whereas patients in GOLD groups $\mathrm{C}$ and $\mathrm{D}$ had more exacerbations at baseline than during follow-up. From baseline to follow-up, the exacerbation rate doubled for GOLD groups A and B - driven by an increase in severe exacerbations, whereas both the moderate and the severe exacerbation rates were lower in GOLD group C and D in the same period. Both moderate and severe exacerbations were more evenly distributed across GOLD groups during the follow-up year (Table 2). 
Table I Baseline Characteristics of the COPD Cohort by GOLD Group A-D and for the Total Cohort of 49,826 COPD Patients

\begin{tabular}{|c|c|c|c|c|c|}
\hline & Gold A & Gold B & Gold C & Gold D & All \\
\hline Patients, $\mathrm{n}$ & 13,266 & 16,415 & 5,790 & 14,355 & 49,826 \\
\hline \multicolumn{6}{|l|}{ Gender, \% } \\
\hline Female & 48.2 & 51.1 & 54.7 & 55.8 & 52.1 \\
\hline \multicolumn{6}{|l|}{ Cohabitation status, \% } \\
\hline Cohabitation & 59.4 & 50.5 & 51.3 & 45.7 & 51.6 \\
\hline Living alone & 40.6 & 49.5 & 48.7 & 54.3 & 48.4 \\
\hline Age, mean (SD) & $65.89(10.20)$ & $69.91(10.12)$ & $68.49(10.32)$ & $71.64(9.73)$ & $69.17(10.29)$ \\
\hline \multicolumn{6}{|l|}{ Age group (years), \% } \\
\hline $40-49$ & 6.4 & 3.0 & 4.0 & 1.9 & 3.7 \\
\hline $50-59$ & 20.7 & 13.5 & 15.8 & 10.3 & 14.8 \\
\hline $60-69$ & 35.5 & 29.0 & 31.7 & 26.2 & 30.2 \\
\hline $70-79$ & 28.0 & 36.5 & 33.2 & 38.9 & 34.6 \\
\hline $80+$ & 9.3 & 18.1 & 15.2 & 22.7 & 16.7 \\
\hline Moderate exacerbations, mean (SD) & $0.10(0.30)$ & $0.15(0.35)$ & $0.35(0.82)$ & $0.46(0.92)$ & $0.25(0.64)$ \\
\hline \multicolumn{6}{|l|}{ Moderate exacerbations, \% } \\
\hline 0 & 90.0 & 85.4 & 80.7 & 74.1 & 82.8 \\
\hline $\mathrm{I}-2$ & 10.0 & 14.6 & 15.9 & 21.3 & 15.4 \\
\hline $3+$ & 0.0 & 0.0 & 3.5 & 4.6 & 1.7 \\
\hline Severe exacerbations, mean (SD) & $0.00(0.00)$ & $0.00(0.00)$ & $1.06(0.52)$ & I.2I (0.72) & $0.47(0.72)$ \\
\hline \multicolumn{6}{|l|}{ Severe exacerbations, $\%$} \\
\hline 0 & 100.0 & 100.0 & 7.2 & 7.5 & 62.6 \\
\hline $1-2$ & 0.0 & 0.0 & 90.7 & 86.8 & 35.5 \\
\hline $3+$ & 0.0 & 0.0 & 2.1 & 5.8 & 1.9 \\
\hline \multicolumn{6}{|l|}{ MRC score, \% } \\
\hline 1 & 28.1 & 0.0 & 22.8 & 0.0 & 10.1 \\
\hline 2 & 71.9 & 0.0 & 77.2 & 0.0 & 28.1 \\
\hline 3 & 0.0 & 54.6 & 0.0 & 43.2 & 30.4 \\
\hline 4 & 0.0 & 30.7 & 0.0 & 33.8 & 19.8 \\
\hline 5 & 0.0 & 14.7 & 0.0 & 23.0 & 11.5 \\
\hline Death during follow up (I Year), \% & 3.2 & II.I & 6.1 & 17.4 & 10.2 \\
\hline
\end{tabular}

\section{Direct Cost, Elderly Care Cost, and Indirect Costs}

Total costs, including direct costs of COPD and cost for elderly care and retirement home, were higher for the COPD patients $(€ 28,969)$ compared with the reference population (€10,693), see Table 3. The same pattern was seen for all GOLD groups and their individually matched comparisons. Direct costs for the COPD population were driven largely by higher costs associated with hospitalizations, as well as costs for outpatient visits and prescription drugs. The indirect costs, defined as foregone earnings, were $€ 6,773$ for the total COPD population.
The stratification by GOLD groups showed that the direct costs were lowest in GOLD A, highest in group GOLD D. Approximately in between GOLD A and GOLD $\mathrm{D}$ and fairly equal came GOLD B and GOLD C (Table 3). The retirement home costs were substantial, and higher in patients from GOLD B compared to patients from GOLD C. The foregone earnings were highest in GOLD B.

To investigate the effect of respiratory infections on total direct and elderly costs, antibiotics were included in the definition of baseline exacerbations. However, it did not change the mean annual direct costs to any notable extent (total direct and elderly costs incl. antibiotics: 
Table 2 Mean Number of Moderate and Severe Exacerbations During the Baseline Year and During the Follow-Up Year, by GOLD Group

\begin{tabular}{|l|c|c|c|c|}
\hline \multirow{2}{*}{} & \multicolumn{2}{|c|}{ Baseline } & \multicolumn{2}{c|}{ Follow-Up } \\
\cline { 2 - 5 } & Moderate Exacerbations & Severe Exacerbations & Moderate Exacerbations & Severe Exacerbations \\
\hline GOLD A & 0.10 & 0.00 & 0.10 & 0.07 \\
\hline GOLD B & 0.15 & 0.00 & 0.16 & 0.21 \\
\hline GOLD C & 0.35 & 1.06 & 0.26 & 0.28 \\
\hline GOLD D & 0.46 & 1.21 & 0.31 & 0.57 \\
\hline Total & $\mathbf{0 . 2 5}$ & $\mathbf{0 . 4 7}$ & $\mathbf{0 . 2 0}$ & $\mathbf{0 . 2 9}$ \\
\hline
\end{tabular}

GOLD A €8,324; GOLD B €12,295; GOLD C €10,702; and GOLD D $€ 16,930$ ).

A major driver of direct costs was the cost of severe exacerbations. The mean costs per moderate and severe exacerbation were $€ 888$ and $€ 7,091$, respectively (Table 4). The costs of an exacerbation (both moderate and severe) did not differ substantially between GOLD groups.

\section{COPD and Non-COPD-Related Costs}

The costs for non-COPD-related HCRU were higher than the COPD-related costs in all GOLD groups except GOLD $\mathrm{D}$ (see Figure 2). The distribution of COPD-related and non-COPD-related costs was approximately the same in GOLD group $\mathrm{B}$ and $\mathrm{C}$. The greatest cost driver in all GOLD groups was hospitalizations, both COPD-related and all causes.

\section{Discussion}

This nationwide population-based cohort study has provided a precise insight into the economic consequences of COPD in Denmark, using a large sample of high-quality registry data.

We found that direct costs were considerably higher among COPD patients compared with the reference population, with an approximate threefold difference in total direct and elderly care costs between the two populations. Direct costs for the COPD population were largely driven by higher costs associated with hospitalizations, whereas outpatient visits and prescription of drugs accounted for a smaller part. Interestingly, costs of an exacerbation (both moderate and severe) did not differ substantially between GOLD groups.

The current findings support previous studies that have reported higher direct costs with increasing severity of disease, as summarized in a recent review from 2020, including European studies ${ }^{21}$ and from 2019 from across the globe (Europe, USA and Asia), ${ }^{22}$ by ur Rehman et al. These reviews highlight various contributing factors to the rising cost of COPD management, including disease severity and the frequency of exacerbations. Also, hospitalizations as well as costs for outpatient visits and prescription drugs are often found to be main drivers of costs - especially in the western world. ${ }^{21-23}$

Most of the studies mentioned above have been conducted in a hospital setting. However, in a large retrospective cohort study from General Practice in the UK it was also demonstrated that costs increased with number of exacerbations. $^{24}$

Two smaller studies have revealed increasing symptom burden to be associated with increasing HCRU. ${ }^{25,26}$

Our data set included symptom measures, which enabled a cost analysis based on GOLD groups. This definition of COPD severity is unique and the findings are therefore not directly comparable with previous results. Our study shows that the direct costs are lowest and highest in GOLD group $\mathrm{A}$ and $\mathrm{D}$, respectively, but that the costs in both GOLD group B and C are approximately in between group $\mathrm{A}$ and $\mathrm{D}$ and fairly equal. This indicates that symptom burden in addition to exacerbation frequency is also important from a cost perspective, thus budgetary concerns should not focus on exacerbations only.

Direct comparison between studies from different geographical areas is difficult due to differences in health-care and reimbursement systems across continents and even between countries. Study designs with matched control populations have the advantage to level out some of the issues related to these differences, which theoretically makes comparisons of findings across regions and countries more reliable. However, only few studies have used 


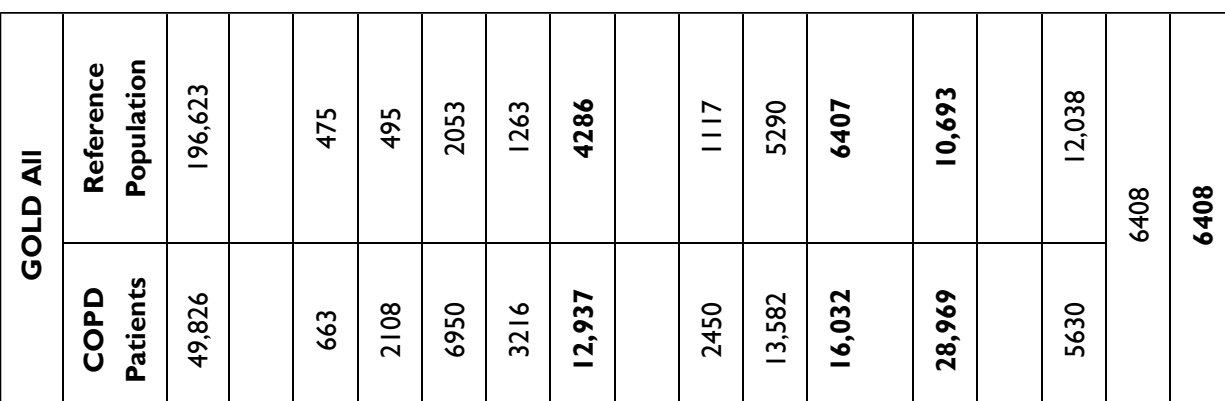

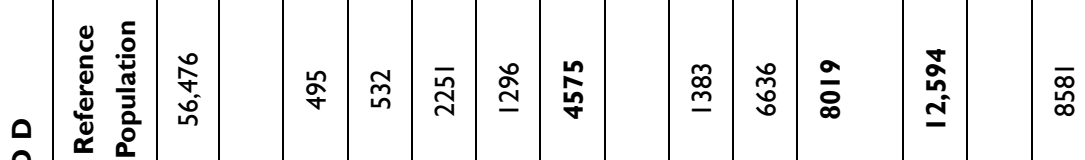

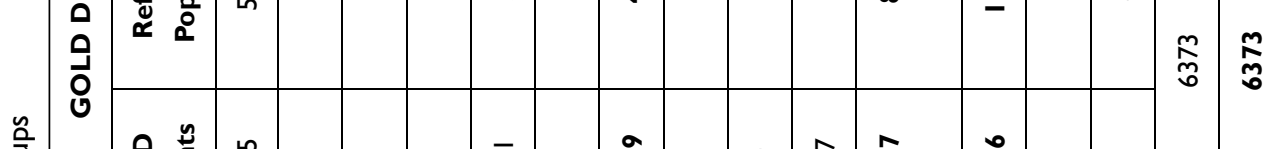


Table 4 Number of Moderate and Severe Exacerbations and Mean Cost (in Euro [€]) of Exacerbation (Counting 28-Days from Start) per Patient by GOLD Group

\begin{tabular}{|l|c|c|c|c|c|c|c|}
\hline \multirow{2}{*}{$\begin{array}{l}\text { GOLD } \\
\text { Group }\end{array}$} & $\begin{array}{c}\text { Patients } \\
(\mathbf{n})\end{array}$ & \multicolumn{3}{|c|}{ Moderate Exacerbation } & \multicolumn{3}{c|}{ Severe Exacerbation } \\
\cline { 3 - 8 } & $\begin{array}{c}\text { Number of } \\
\text { Exacerbations }\end{array}$ & $\begin{array}{c}\text { \% of Patients } \\
\text { Experiencing } \\
\text { Exacerbation }\end{array}$ & $\begin{array}{c}\text { Mean Cost per } \\
\text { Exacerbation per } \\
\text { Patient (€) }\end{array}$ & $\begin{array}{c}\text { Number of } \\
\text { Exacerbations }\end{array}$ & $\begin{array}{c}\text { \% of } \\
\text { Patients }\end{array}$ & $\begin{array}{c}\text { Mean Cost per } \\
\text { Exacerbation per } \\
\text { Patient (€) }\end{array}$ \\
\hline GOLD A & 13,266 & 1273 & 9.6 & 744 & 971 & 7.32 & 6616 \\
\hline GOLD B & 16,415 & 2624 & 16.0 & 962 & 3408 & 20.76 & 7083 \\
\hline GOLD C & 5790 & 1510 & 26.1 & 791 & 1645 & 28.41 & 6928 \\
\hline GOLD D & 14,355 & 4513 & 31.4 & 917 & 8212 & 57.21 & 7182 \\
\hline Total & $\mathbf{4 9 , 8 2 6}$ & $\mathbf{9 9 2 0}$ & $\mathbf{1 9 . 9 1}$ & $\mathbf{8 8 8}$ & 14,236 & $\mathbf{2 8 . 5 7}$ & $\mathbf{7 0 9 1}$ \\
\hline
\end{tabular}

population-based, matched controls. Furthermore, data completeness as well as the available economic parameters differ.

A number of studies from around the world have reported that COPD is a significant societal economic burden, associated with increased HCRU and higher health care costs. ${ }^{23,27-29}$ Additionally, higher direct costs (including mean costs per patient per year and mean costs per exacerbation) seem to be associated with increasingly severe COPD and/or a history of more frequent or severe exacerbations. In our study, indirect costs also contributed significantly to the combined costs. This was also reported in study from Germany, ${ }^{30}$ that found COPD to be significantly associated with excess direct and indirect costs that both increased with disease severity. However, indirect costs are largely unavailable in most other studies.
In the study from Sweden by Lisspers et $\mathrm{al}^{8}$ the price of a moderate and severe exacerbation seemed in line with the finding from our study, although they found severe exacerbations to be more expensive for patients in GOLD group D. This latter finding is most likely due to small numbers and to the fact that average exacerbation costs for moderate and severe exacerbations were calculated based on HCRU during 14 days after the occurrence of an exacerbation event - we used 28 days. The shorter the observation period, the length of the hospital stay comes to weights in relation to the total expenses.

As the prevalence of comorbidities in patients with COPD is high, it is important to understand the impact of costs for comorbidities in this population. We showed that for GOLD group A-C the non-COPD-related direct costs were higher than the COPD-related cost. Prevention of severe comorbidities and managing them more

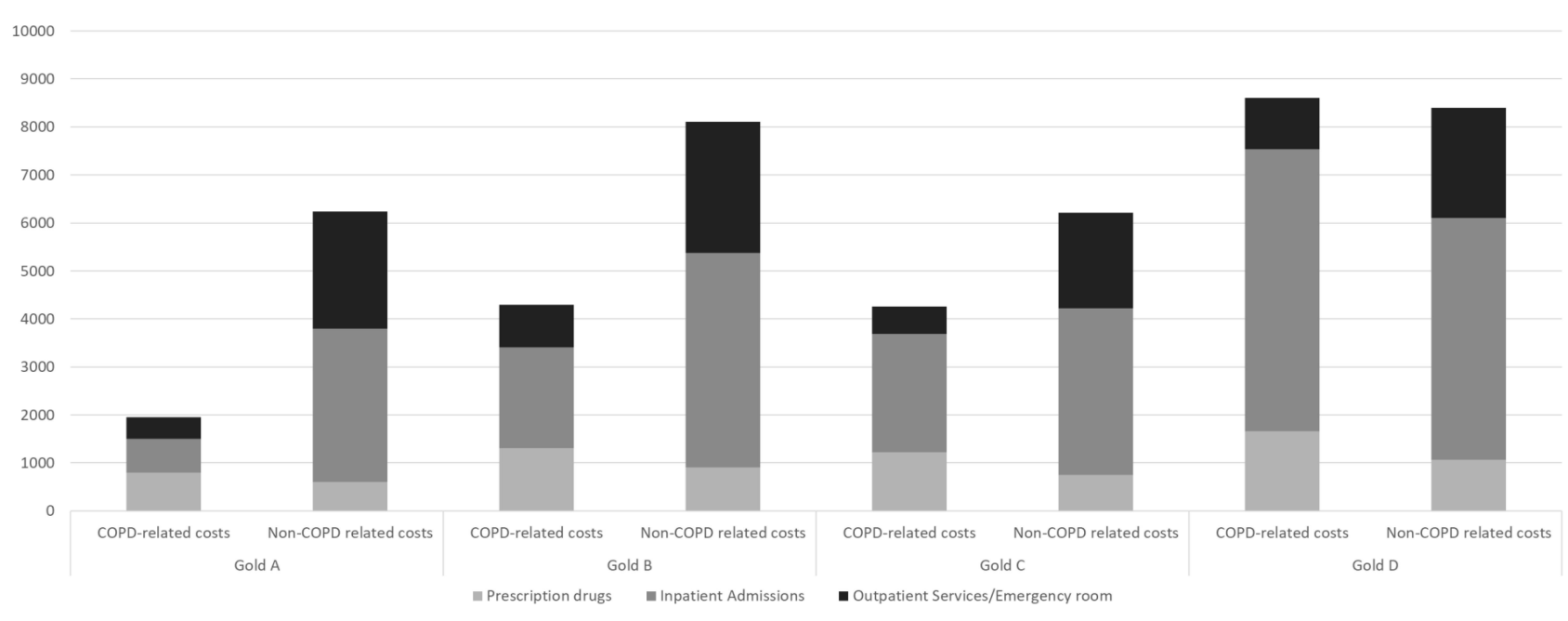

Figure 2 Direct costs in euro $(€)$ in COPD patients, presented as COPD-related and non-COPD-related costs and stratified by GOLD group. 
effectively would not only be of benefit for the patients but could potentially also relieve much of the financial burden of disease. This has been demonstrated in a recent study, evaluating the cost analysis of COPD exacerbations and cardiovascular events in the SUMMIT trial. ${ }^{31}$

The main strengths in our study are the large sample size, covering patients across the entire COPD severity spectrum, and the use of matched population comparisons to reflect healthcare use and cost in individuals from the general population of same age, sex and cohabitation status. The availability of dyspnea symptom scores in combination with the comprehensive range of costs measured, and the extended assessment period generated robust economic data for patients with COPD and a matched reference population across time, age, and disease stage. Finally, this study has recognized the importance of indirect costs in the economic burden of COPD on the Danish society. However, when interpreting the results, one should be aware that patients included in this study were identified in a hospital setting. Thus, the present study reflects a more severe COPD population than if identified in the general population. This would intuitively result in higher cost estimates. In contrast, patients in the control group may have been treated for COPD in primary care, which to some extend likely lowered our estimate of the cost difference. Furthermore, information on smoking was not available in the nationwide population registers, that were used for matching COPD patients with comparisons from the general population. Most of our analyses focus on analysis within the COPD cohort only and are therefore not expected to be influenced. But the analysis comparing mean annual costs between COPD patients and the reference population has been affected as it measured both the cost of COPD and the cost of smoking-related illness in the COPD cohort which has much higher smoking rates than the general population.

We found the yearly exacerbation rate doubled for GOLD groups A and B during follow-up - driven by an increase in severe exacerbations whereas both the moderate and the severe exacerbation rates were lower in GOLD group $\mathrm{C}$ and $\mathrm{D}$ in the same period.

The latter finding most likely reflects the regression towards the mean and a direct consequence of the high mortality rates in both GOLD group C and D during follow-up.

The increase in severe exacerbations in GOLD groups $\mathrm{A}$ and $\mathrm{B}$ during follow-up points towards the importance of earlier diagnosis and management of COPD, as this would not only benefit patients, but hopefully also reduce the economic burden of disease by interrupting the progression of mild and moderate patients with COPD to more severe stages of disease.

\section{Conclusion}

In this large real-world study, we showed that total direct and indirect costs were approximately threefold higher among patients with COPD compared to a matched reference population. Direct costs for the COPD population were largely driven by higher costs associated with hospitalizations as well as costs for outpatient visits and prescription drugs. The costs increased with increasing disease severity, as assessed by GOLD A-D grouping, reflecting previous exacerbation history and daily symptom burden. Notably, costs of a moderate exacerbation did not differ substantially across GOLD groups and the same was seen for severe exacerbations.

\section{Data Sharing Statement}

The dataset supporting the conclusions of this article can be available upon request.

\section{Ethics Approval and Consent to Participate}

This study was performed in accordance with the Declaration of Helsinki, the International Council for Harmonisation of Technical Requirements for Pharmaceuticals for Human Use Good Clinical Practices, Good Pharmacoepidemiology Practice and the applicable legislation on non-interventional studies and/or observational Studies. All data accessed complied with relevant data protection and privacy regulations.

\section{Consent for Publication}

All authors made a significant contribution to the work reported, whether that is in the conception, study design, execution, acquisition of data, analysis and interpretation, or in all these areas; took part in drafting, revising or critically reviewing the article; gave final approval of the version to be published; have agreed on the journal to which the article has been submitted; and agree to be accountable for all aspects of the work.

\section{Funding}

This study was sponsored by AstraZeneca. 


\section{Disclosure}

A.L., J.L., R.I., and O.H. report no conflict of interests in relations to the current study. P.L. has participated in advisory boards for AstraZeneca, Boehringer Ingelheim, Chiesi and GSK, and has received research grants from AstraZeneca, Boehringer Ingelheim and GSK. M.A. and S. dFL. are full-time employees of AstraZeneca. The authors report no other conflicts of interest in this work.

\section{References}

1. ERS - Respiratory health and disease in Europe. Available from: https://www.erswhitebook.org/. Accessed June 21, 2020.

2. Forum of International Respiratory Societies. The Global Impact of Respiratory Disease. 2nd ed. Sheffield: European Respiratory Society, Forum of International Respiratory Societies; 2017.

3. Guarascio AJ, Ray SM, Finch CK, Self TH. The clinical and economic burden of chronic obstructive pulmonary disease in the USA. Clin Outcomes Res. 2013;5:235-245.

4. Løkke A, Fabricius PG, Vestbo J, Marott JL, Lange P. Prevalence of chronic obstructive pulmonary disease in Copenhagen. Results from the Copenhagen city heart study. Ugeskr Lager. 2007;169(46): 3956-3960.

5. Bilde L, Rud Svenning A, Dollerup J, Bække Borgeskov H, Lange P. The cost of treating patients with COPD in Denmark - A population study of COPD patients compared with non-COPD controls. Respir Med. 2007;101:539-546. doi:10.1016/j.rmed.2006.06.020

6. Løkke A, Hilberg O, Tønnesen P, et al. Direct and indirect economic and health consequences of COPD in Denmark: a national registerbased study: 1998-2010. BMJ Open. 2014;4:e004069. doi:10.1136/ bmjopen-2013-004069

7. Løkke A, Hilberg O, Kjellberg J, Ibsen R, Jennum P. Economic and health consequences of COPD patients and their spouses in Denmark-1998-2010. COPD J Chronic Obstr Pulm Dis. 2014;11: 237-246.

8. Lisspers K, Larsson K, Johansson G, et al. Economic burden of COPD in a Swedish cohort: the ARCTIC study. Int $J$ COPD. 2018;13:275-285. doi:10.2147/COPD.S149633

9. Dalal AA, Patel J, D’Souza A, Farrelly E, Nagar S, Shah MJ. Impact of COPD exacerbation frequency on costs for a managed care population. Manag Care Spec Pharm. 2015;21(7):575-583.

10. Byng D, Lutter JI, Wacker ME, et al. Determinants of healthcare utilization and costs in COPD patients: first longitudinal results from the German COPD cohort COSYCONET. Int J Chron Obstruct Pulmon Dis. 2019;14:1423-1439. doi:10.2147/COPD.S201899

11. Foo J, Landis SH, Maskell J, et al. Continuing to confront COPD international patient survey: economic impact of COPD in 12 countries. PLoS One. 2016;11(4):e0152618. doi:10.1371/journal. pone. 0152618

12. Chen W, FitzGerald JM, Sin DD, Sadatsafavi M. Excess economic burden of comorbidities in COPD: a 15-year population-based study. Eur Respir J. 2017;50:1-10. doi:10.1183/13993003.00393-2017

13. Global Initiative for Chronic Obstructive Lung Disease - GOLD. Available from: https://goldcopd.org/. Accessed June 21, 2020.

14. Schmidt M, Schmidt SA, Sandegaard JL, Ehrenstein V, Pedersen L, Sorensen HT. The Danish National Patient Registry: a review of content, data quality, and research potential. Clin Epidemiol. 2015;7:449-490. doi:10.2147/CLEP.S91125
15. Lange P, Tottenborg SS, Sorknaes AD, et al. Danish Register of chronic obstructive pulmonary disease. Clin Epidemiol. 2016;8: 673-678. doi:10.2147/CLEP.S99489

16. Pottegard A, Schmidt SAJ, Wallach-Kildemoes H, Sorensen HT, Hallas J, Schmidt M. Data resource profile: the Danish national prescription registry. Int J Epidemiol. 2017;46(3):798-f. doi:10. 1093/ije/dyw213

17. Baadsgaard M, Quitzau J. Danish registers on personal income and transfer payments. Scand J Public Health. 2011;39(7 Suppl):1 03-105. doi:10.1177/1403494811405098

18. Schmidt M, Pedersen L, Sorensen HT. The Danish civil registration system as a tool in epidemiology. Eur J Epidemiol. 2014;29 (8):541-549. doi:10.1007/s10654-014-9930-3

19. Stenton C. The MRC breathlessness scale. Occup Med (Chic Ill). 2008;58:226-227. doi:10.1093/occmed/kqm162

20. Blough DK, Ramsey SD. Using generalized linear models to assess medical care costs. Health Serv Outcomes Res Methodol. 2000;1 (2):185-202. doi:10.1023/A:1012597123667

21. ur Rehman A, Hassali MAA, Muhammad SA, Harun SN, Shah S, Abbas S. The economic burden of chronic obstructive pulmonary disease (COPD) in Europe: results from a systematic review of the literature. Eur J Health Econ. 2020;21(2):181-194. doi:10.1007/ s10198-019-01119-1

22. ur Rehman A, Hassali MAA, Muhammad SA, et al. The economic burden of chronic obstructive pulmonary disease (COPD) in the USA, Europe, and Asia: results from a systematic review of the literature. Expert Rev Pharmacoecon Outcomes Res. 2020;20:661-672.

23. Iheanacho I, Zhang S, King D, Rizzo M, Ismaila AS. Economic burden of chronic obstructive pulmonary disease (COPD): a systematic literature review. Int $J$ Chron Obstruct Pulmon Dis. 2020;26(15):439-460. doi:10.2147/COPD.S234942

24. Punekar YS, Shukla A, Müllerova H. COPD management costs according to the frequency of COPD exacerbations in UK primary care. Int J Chron Obstruct Pulmon Dis. 2014;9:65-73. doi:10.2147/ COPD.S54417

25. Ding B, Small M, Bergström G, Holmgren U. COPD symptom burden: impact on health care resource utilization, and work and activity impairment. Int J Chron Obstruct Pulmon Dis. 2017;21 (12):677-689. doi:10.2147/COPD.S123896

26. Stephenson JJ, Wertz D, Gu T, Patel J, Dalal AA. Clinical and economic burden of dyspnea and other COPD symptoms in a managed care setting. Int J Chron Obstruct Pulmon Dis. 2017;4 (12):1947-1959. doi:10.2147/COPD.S134618

27. Souliotis K, Kousoulakou H, Hillas G, Tzanakis N, Toumbis M, Vassilakopoulos $\mathrm{T}$. The direct and indirect costs of managing chronic obstructive pulmonary disease in Greece. Int $J$ Chron Obstruct Pulmon Dis. 2017;12:1395-1400. doi:10.2147/COPD.S132825

28. Wallace AE, Kaila S, Bayer V, et al. Health care resource utilization and exacerbation rates in patients with COPD stratified by disease severity in a commercially insured population. J Manag Care Spec Pharm. 2019;25(2):205-217. doi:10.18553/jmcp.2019.25.2.205

29. Liang L, Shang Y, Xie W, Shi J, Tong Z, Jalali MS. Trends in hospitalization expenditures for acute exacerbations of COPD in Beijing from 2009 to 2017. Int J Chron Obstruct Pulmon Dis. 2020;15:1165-1175. doi:10.2147/COPD.S243595

30. Wacker ME, Jörres RA, Schulz H, et al.; COSYCONET-Consortium. Direct and indirect costs of COPD and its comorbidities: results from the German COSYCONET study. Respir Med. 2016;111:39-46. doi:10.1016/j.rmed.2015.12.001

31. Stanford RH, Coutinho AD, Eaddy M, Yue B, Bogart M. Cost analysis of COPD exacerbations and cardiovascular events in SUMMIT. Am J Manag Care. 2019;25(11):e320-e325. 


\section{Publish your work in this journal}

The International Journal of COPD is an international, peer-reviewed journal of therapeutics and pharmacology focusing on concise rapid reporting of clinical studies and reviews in COPD. Special focus is given to the pathophysiological processes underlying the disease, intervention programs, patient focused education, and self management protocols. This journal is indexed on PubMed Central, MedLine and CAS. The manuscript management system is completely online and includes a very quick and fair peer-review system, which is all easy to use. Visit http://www.dovepress.com/testimonials.php to read real quotes from published authors. 